RESEARCH

Turkish Journal of Geriatrics

DOI: 10.31086/tigeri.2021.253

2021; 24(4): 557-566

\title{
THE ASSOCIATION BETWEEN PROGNOSIS AND SARCOPENIA ASSESSED BY PSOAS MUSCLE MEASUREMENTS IN ELDERLY MALE PATIENTS WITH COVID-19
}

- Musa POLAT ${ }^{1,6}$

- Çiğdem Samur SALBAŞ²

- Sema SARI ${ }^{3}$

- Mustafa DOĞAN ${ }^{4}$

- Selim ÇAM ${ }^{5}$

-Ahmet KARADAĞ6

CORRESPONDANCE

\section{${ }^{6}$ Musa POLAT}

Sivas Cumhuriyet University, Physical Medicine and Rehabilitation, Sivas, Turkey

\section{Phone: +905058634671}

e-mail: musa.polat.sf@gmail.com

Received: Aug 19, 2021

Accepted: Nov 22, 2021

${ }^{1}$ Niğde Omer Halisdemir University, Physical Medicine and Rehabilitation, Niğde, Turkey

${ }^{2}$ Niğde Omer Halisdemir University Training and Research Hospital, Radiology, Niğde, Turkey

${ }^{3}$ Niğde Omer Halisdemir University Training and Research Hospital, Infectious Diseases, Niğde, Turkey

${ }^{4}$ Nigde Omer Halisdemir University, Forensic Medicine, Niğde, Turkey

${ }^{5}$ Sivas Cumhuriyet University, Hospital Statistic, Sivas, Turkey

6 Sivas Cumhuriyet University, Physical Medicine and Rehabilitation, Sivas, Turkey

\section{Abstract}

Introduction: Sarcopenia, which is defined as a generalized loss of skeletal muscle mass and function, is an important clinical condition, especially among elderly patients with comorbidities. This study assesses the association between prognosis and sarcopenia assessed by psoas muscle measurements in elderly male patients with coronavirus disease 2019.

Materials and Methods: This study included 130 consecutive elderly male patients hospitalized with coronavirus disease 2019. The participants were followed until their time of discharge or death. The psoas muscle crosssectional area, psoas density, and psoas muscle index measurements were performed based on a single-axial computed tomography section at the $\mathrm{L} 2$ vertebral corpus. The outcome measurements of the patients were determined as intensive care unit admission and mortality.

Results: During the clinical course of these patients, 29.2\% (38) were transferred to the intensive care unit, and $13.1 \%$ (17) died. The interclass correlation coefficients for the psoas muscle cross-sectional area and density were 0.91 and 0.93 , respectively, between observers. Low psoas muscle index is significantly associated with mortality $(p=0.001)$, and the probability of mortality increases approximately 5-30 times as the psoas muscle index decreases. No factors were identified as being significantly associated with intensive care unit admission.

Conclusion: In elderly male patients who are hospitalized due to coronavirus disease 2019, an assessment of the psoas muscle may provide additional information on the prognosis of the disease.

Keywords: Sarcopenia; COVID-19; Intensive Care Units; Mortality. 


\section{INTRODUCTION}

The SARS-COV-2 virus that causes coronavirus disease 2019 (COVID-19) has continued to spread for more than a year and has led to significant social, economic, and political problems, bringing about a significant increase in mortality and morbidity rates, as with other pandemics. More than 126 million people worldwide had been diagnosed with COVID-19, and nearly 3 million people had died from COVID-19-related causes as of March 29, 2021 (1). These numbers are a strong indicator of the magnitude of the pandemic and its threat to current healthcare systems due to limited resources.

During pandemics, limited access to healthcare resources requires appropriate triage, and for both appropriate triage and successful patient management, prognostic factors should be well known (2). Previous studies have found advanced age, male gender, high Sequential Organ Failure Assessment Scores, and high D-dimer levels and comorbidities to be associated with poor prognosis in COVID-19 patients $(3,4)$. In addition, a recent meta-analysis found that frailty increases the severity, morbidity, and mortality risk associated with COVID-19 (2). Several guidelines, such as the National Institute for Health and Care Excellence guidelines, can be recommended for the assessment of frailty in COVID-19 patients over the age of 65 years (5). Frailty is associated with several clinical conditions, but it is often accompanied by sarcopenia (6). Defined as a generalized loss of skeletal muscle mass and function, sarcopenia is an important clinical condition, especially among elderly patients with comorbidities (7), because it affects not only motor activity but also respiratory function, the immune system, and the ability of an individual to cope with metabolic stress (8).

Many radiological and anthropometric measurement methods have been used to measure skeletal muscle mass as a sarcopenia assessment method, including dual-energy X-ray absorptiometry, computed tomography (CT), and magnetic resonance imaging. Although muscle mass measurements that are taken from whole-body images yield definite results, the approach is not practical due to its cost, time requirement, and radiation exposure (7). This has led to single-level axial CT and muscle cross-sectional area (CSA) measurement techniques being applied in previous studies. The high inter-observer agreement of CSA measurements increases their reliability (9). It has been observed that the CSA measurements of various muscles, such as the psoas muscle, the paravertebral muscles, and the thigh muscles, performed using this method reflect overall body muscle mass (7).

Psoas muscle CSA or psoas muscle index (PMI) measurements from CT images have been shown to have prognostic value in various diseases (9-12). Some studies have reported a relationship between the psoas muscle CSA and the duration of ventilator weaning and mortality in elderly intensive care patients and hospital mortality in pulmonary embolism patients monitored in the intensive care unit (ICU) (11-12). However, to the best of our knowledge, there have been no studies to date evaluating the association between psoas muscle measurements and the course of disease in elderly COVID-19 patients. Accordingly, the present study assesses the association between psoas muscle CSA, psoas density, and PMI measurements and ICU admission and mortality in elderly male patients hospitalized with COVID-19.

\section{MATERIALS AND METHODS}

\section{Study population}

This study included 130 consecutive elderly male patients over 65 years of age who were diagnosed with COVID-19 via a real-time reverse transcription-polymerase chain reaction test on nasopharyngeal/oropharyngeal swab samples. The patients were hospitalized in accordance with the Turkish Ministry of Health COVID-19 Patient Management algorithm between May 1, 2020 and July 30, 2020 
in a training and research hospital that is a tertiary care referral hospital in Turkey (13). Patients with a history of ICU stay for any reason, a history of spinal surgery, artifacts on CT images that could affect the analysis, or scoliosis were excluded from the study. The study patients were followed up until their time of discharge or death. The patients who were transferred to the ICU during follow-up were included in the ICU group, and patients who died during follow-up either on the ward or in the ICU were included in the mortality group. Local ethics committee approval was obtained prior to undertaking the study (E-95860085-050.02.04-19842-2020/75). The participants were provided with written and verbal information, and all provided their consent. This study was conducted in accordance with the Declaration of Helsinki.

\section{Clinical data and outcome measures}

The age, body mass index (BMI), and comorbidities of the study participants were recorded. The time from the onset of symptoms at admission, body temperature measurements, respiratory rate per minute, oxygen saturation in room air, $\mathrm{PaO} 2 /$ $\mathrm{FiO} 2$, and laboratory values (creatinine, leukocyte count, lymphocyte count, hemoglobin, platelet count, ALT, AST, LDH, CK, ferritin, CRP, procalcitonin, D-dimer, and PT) were recorded. The outcome measurements of the patients who were followed up until discharge or death were determined as ICU admission and mortality.

The above-mentioned algorithm recommends the performance of thoracic $\mathrm{CT}$ on patients with at least one of the following findings: respiratory distress, low oxygen saturation in room air (SpO2 $<93 \%$ ), a respiratory rate per minute $\geq 24$, and the presence of other pathological findings upon respiratory system examination. The algorithm recommends hospitalization for patients with poor prognostic criteria on blood tests (blood lymphocyte count 10x the upper limit of normal, ferritin $>500$ $\mathrm{ng} / \mathrm{ml}$, or D-dimer $>1,000 \mathrm{ng} / \mathrm{ml}$, etc.), a respiratory rate $>24$ /minute, $\mathrm{SpO} 2$ in room air $<93 \%$, or bilateral diffuse (> 50\%) involvement on lung imaging. It further suggests that upon hospitalization or follow-up, patients can be followed in the ICU if they have any of the following symptoms (13):

- Dyspnea and respiratory distress, a respiratory rate $\geq 30 / \mathrm{min}$, and $\mathrm{PaO} 2 / \mathrm{FiO} 2<300$, despite current treatment,

- Increased need for oxygen at follow-up

- $\mathrm{SpO} 2<90 \%$ or $\mathrm{PaO} 2<70 \mathrm{mmHg}$, despite 5 L/min oxygen therapy

- Hypotension (systolic blood pressure $<90$ $\mathrm{mmHg}$ or a drop in normal systolic blood pressure greater than $40 \mathrm{mmHg}$ and mean arterial pressure $<65 \mathrm{mmHg}$, tachycardia $>$ $100 /$ min)

- Development of acute organ dysfunction, such as acute kidney injury, any disorder revealed in acute liver function tests, confusion, acute hemorrhagic diathesis, and immunosuppression

- Elevated troponin and arrhythmia

- Lactate $>2 \mathrm{mmol}$

- Impaired capillary refill

- Skin disorders, such as cutis marmorata

\section{Computed tomography image analysis and psoas muscle measurements}

$\mathrm{CT}$ examinations were conducted using the images acquired at the time of the initial hospital admission. The images were numbered, and two different sort orders were created using a computer program. The first order involved the assessment of parenchymal involvement on the thoracic CT images, while the second order included measurements of the psoas muscle. The thoracic CT images were classified by the radiologist as consolidation, ground-glass appearance, and bilateral diffuse infiltration depending on the type of parenchymal in- 
volvement (14). Psoas muscle measurements were performed independently and manually by a radiologist and a physiatrist who were blinded to the clinical and laboratory findings of the patients based on a single axial section at the $\mathrm{L} 2$ vertebral corpus using the previously published technique (15). The psoas muscle boundaries were determined using the specific Hounsfield unit threshold of -29 to +150 for skeletal muscle. The psoas muscle CSA was measured separately for the right and left sides using the Karmed PACS (Kardelen Software, Turkey) program (Figure 1), with three consecutive measurements conducted for the right and left psoas muscles, which were then averaged. The mean psoas muscle CSA was obtained by summing the values obtained during the separate measurement of the psoas muscle CSA on both sides and dividing by two. To normalize the CSA measurement, the mean psoas muscle CSA was divided by the BMI, yielding the PMI (16). Psoas density was measured using the Hounsfield unit average calculation approach with the following formula (17):

Hounsfield unit average calculation $=[($ right mean psoas Hounsfield unit density $X$ right CSA) + (left mean psoas Hounsfield unit density $X$ left psoas CSA)] / (Total CSA)

Figure 1. Bilateral psoas muscle cross-sectional area measurement in a single axial computed tomography section at the L2 vertebral corpus

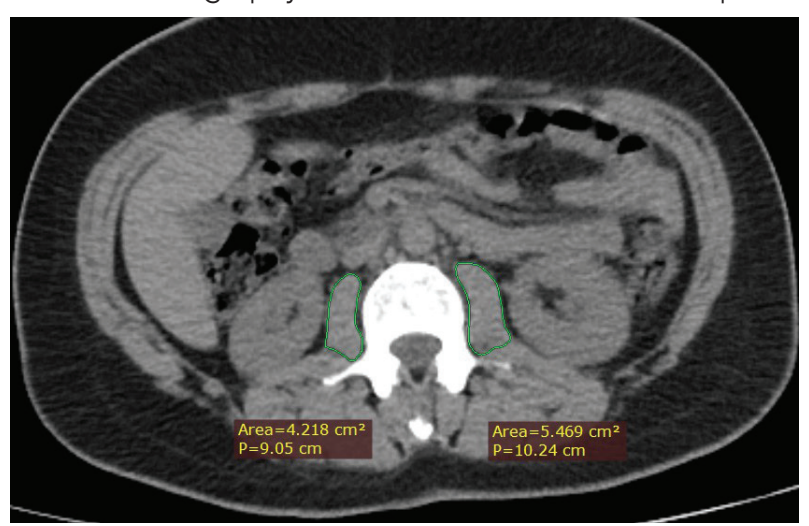

\section{Statistical analysis}

This study used the baseline data upon patient admission, and statistical analyses were performed using IBM SPSS Statistics (Version 22.0. Armonk, NY: IBM Corp.). The normality of the variables was analyzed using visual (histogram and probability plots) and analytical methods (Kolmogorov-Smirnov/ Shapiro-Wilk tests). Descriptive values were presented as the median and interquartile range for non-normally distributed variables and frequency and percentage for ordinal and nominal variables. Nominal variables were compared with a Chisquare analysis, while ordinal variables and other clinical, radiological, and laboratory parameters were compared with a Kruskal-Wallis test due to the non-normal distribution. Pairwise comparisons were made with a Mann-Whitney $U$ test and evaluated using the Bonferroni correction. A multinomial logistic regression analysis was used to examine the clinically suspected independent variables that caused either the transfer of patients from the ward to the ICU or death. Outcome measurements, ICU admission, and mortality were used as the dependent variables. A total type- 1 error level of $5 \%$ was used for statistical significance.

\section{RESULTS}

This study included 130 consecutive elderly male patients. The median age was 74 (68-79) years, and the median BMI was $22.4 \mathrm{~kg} / \mathrm{m}^{2}$ (20.5-24.5). Among the participants, $93.8 \%$ (122) had at least one comorbidity, and the most common comorbidities were type 2 diabetes mellitus, hypertension, and chronic obstructive pulmonary disease, with a prevalence of $23.8 \%$ (31), $26 \%(20)$, and $16.9 \%$ (22), respectively. The median time from the onset of symptoms at admission was 6 days (4-8), median body temperature was $37.4^{\circ} \mathrm{C}(36.8-38.6)$, and median oxygen saturation on room air was $88 \%$ (86-89). There was consolidation, a ground glass appearance, and bilateral involvement on thoracic CT images in $27.7 \%$ (36), $35.3 \%$ (46), and $36.9 \%$ (48) of the patients, re- 
spectively. The median psoas muscle CSA was 2.09 $\mathrm{cm}^{2}$ (1.55-3.06), the median PMI was $0.09 \mathrm{~cm}^{2} / \mathrm{kg} /$ $\mathrm{m}^{2}(0.07-0.12)$, and the median psoas density was 49.9 (44.6-53.7) on CT images acquired at the time of initial hospital admission. The demographic, clinical, laboratory, and radiological findings of the participants are presented in Tables 1 and 2. Interobserver agreement was excellent, and the interclass correlation coefficients were 0.91 and 0.93 for the psoas muscle CSA and density, respectively.

During the clinical course of this patient sample, who were treated as inpatients on a pandemic ward upon hospital admission, 29.2\% (38) were transferred to the ICU, 13.1\% (17) died either on the ward or in the ICU, and $57.7 \%$ (75) were on the ward until discharge. All three groups shared similar demographic data, clinical findings, laboratory findings, and lung involvement type (Tables 1 and 2). The psoas muscle CSA and PMI were lower in the non-surviving patients than in the other two groups ( $p<0.001$ for both), while there was no statistically significant difference between the ICU group and the ward group ( $p=0.09, p=0.06)$. The mean psoas densities recorded in the groups were similar ( $p=$ 0.58) (Table 2).

Table 3 presents the results of a multinomial logistic regression analysis conducted to examine the factors associated with the need for intensive care and mortality in COVID-19 patients, showing that PMI, age, and comorbidity are all significantly associated with mortality $(p=0.001, p=0.017$, and $p=0.019$, respectively). The results show that the probability of mortality increases approximately 5-30 times as the PMI decreases and age increases and 1.7-2.6 times due to comorbidity. No factors were identified that were significantly associated with the ICU.

\section{DISCUSSION}

In the present study, we examined the prognostic significance of the psoas muscle CSA, psoas densi- ty, and PMI values for mortality and ICU admission in elderly male patients with COVID-19. The PMI was found to be associated with mortality, while no significant association was identified between PMI and ICU admission for elderly male patients with COVID-19. Furthermore, no association could be established between psoas density and psoas CSA and ICU admission or mortality in elderly male patients with COVID-19. These results are demonstrated for the first time in the literature in the present study.

A low PMI value has been shown to indicate a poor outcome in several acute and chronic clinical conditions (9-12). The present study revealed a relationship between PMI and mortality in COVID-19, which can lead to acute, aggressive, multi-organ failure and mortality. Considering the effects of frailty and sarcopenia, which is one of the most important components of frailty, on both the immune system and metabolic stress and respiratory function, this finding is not surprising $(18,19)$.

Muscle density is believed to reflect muscle quality because it provides information about intramuscular fat storage (20). Although psoas muscle density was shown to be associated with outcome measures of enterocutaneous fistula therapy and certain conditions, such as trauma and gastrointestinal oncological operations, we were unable to establish any association between psoas muscle density and ICU admission and mortality among the hospitalized COVID-19 patients in the present study $(10,17,20-21)$. This result does not necessarily rule out the effect of muscle quality on prognosis because muscle quality is also an important factor in frailty as well as muscle quantity (7). There are many individual factors that could not be evaluated in the present study, such as anatomical structure, biomechanical properties, neuromuscular and metabolic performance, and their effects on muscle quality, which might have led to this result (19). Furthermore, the sample was fairly small and included only elderly male hospitalized COVID-19 patients. Therefore, we may not have reached a sufficient size 
Table 1. Demographic, clinical and laboratory findings of the patients

\begin{tabular}{|c|c|c|c|c|c|}
\hline & $\begin{array}{l}\text { All patients } \\
\quad(n=130)\end{array}$ & $\begin{array}{l}\text { Patients who on } \\
\text { the ward until dis- } \\
\text { charge } \\
(n=75)\end{array}$ & $\begin{array}{l}\text { Patients who trans- } \\
\text { ferred to the ICU } \\
\qquad(n=38)\end{array}$ & $\begin{array}{l}\text { Patients who died } \\
\text { either on the ward } \\
\text { or in the ICU } \\
(n=17)\end{array}$ & $p$ \\
\hline Age, years & $74(68-79)$ & $72(68-79)$ & $74(68-79)$ & $77.3(75-84)$ & 0.14 \\
\hline Sex, male, n(\%) & $130(100)$ & $75(100)$ & $38(100)$ & $17(100)$ & 1 \\
\hline $\mathrm{BMI}, \mathrm{kg} / \mathrm{m}^{2}$ & $22.4(20.5-24.5)$ & $22.6(20.82-24.68)$ & $21.6(20.04-24.27)$ & $22.1(21.2-26.8)$ & 0.75 \\
\hline Symptom Duration, days & $6(4-8)$ & $6(4-8)$ & $6(4-7)$ & $6(5-8)$ & 0.93 \\
\hline Body Temperature, ${ }^{\circ} \mathrm{C}$ & $37.4(36.8-38.6)$ & $37.6(36.8-38.6)$ & $37.5(36.8-38.8)$ & $37.3(36.8-38.2)$ & 0.73 \\
\hline Oxygen Saturation, \% & $88(86-89)$ & $88(84-88)$ & $87(85-89)$ & 88(83-88) & 0.72 \\
\hline Comorbidities & & & & & 0.38 \\
\hline Hypertension & $26(20 \%)$ & $14(18.7 \%)$ & $9(23.7 \%)$ & $3(17.6 \%)$ & \\
\hline Diabetes mellitus & $31(23.8 \%)$ & $18(24 \%)$ & $10(26.3 \%)$ & $3(17.6 \%)$ & \\
\hline COPD & $22(16.9 \%)$ & $13(17.3 \%)$ & $6(15.8 \%)$ & $3(17.6 \%)$ & \\
\hline Malignancy & $7(5.3 \%)$ & $2(2.7 \%)$ & $3(7.9 \%)$ & $2(11.8 \%)$ & \\
\hline Heart failure & $5(3.8 \%)$ & $2(2.7 \%)$ & $2(5.26 \%)$ & $1(5.8 \%)$ & \\
\hline $\begin{array}{l}\text { Cardiovascular } \\
\text { Disease }\end{array}$ & $15(11.5 \%)$ & $11(14.7 \%)$ & $2(5.3 \%)$ & $2(11.8 \%)$ & \\
\hline $\begin{array}{l}\text { Inflammatory } \\
\text { Rheumatic Disease }\end{array}$ & $4(3.1 \%)$ & $1(1.3 \%)$ & $2(5.2 \%)$ & $1(5.8 \%)$ & \\
\hline Chronic renal failure & $8(6.1 \%)$ & $2(2.7 \%)$ & $4(10.5 \%)$ & $2(11.8 \%)$ & \\
\hline Dementia & $3(2.3 \%)$ & $1(1.3 \%)$ & $1(2.6 \%)$ & $1(5.8 \%)$ & \\
\hline \multicolumn{6}{|l|}{ Laboratory Results } \\
\hline Hemoglobin, g/dl & $12.5(11.2-13.8)$ & 12.7(11.2-14.1) & 12.7(11.3-14) & $12.2(11.2-13.8)$ & 0.68 \\
\hline WBC, uL & $8700(5700-11550)$ & $8700(5700-12000)$ & $8750(6400-11400)$ & $9400(5300-11400)$ & 0.63 \\
\hline Lymphocytes, uL & $800(490-1030)$ & $870(540-1030)$ & $785(490-1010)$ & $760(360-980)$ & 0.65 \\
\hline Thrombocyte, uL & $\begin{array}{c}224000(170500- \\
267000)\end{array}$ & $\begin{array}{c}224000(160000- \\
253000)\end{array}$ & $206000(183-270000)$ & $\begin{array}{c}183000(145000- \\
253000)\end{array}$ & 0.75 \\
\hline$A L T, U / L$ & $26(19-36)$ & $26(19-36)$ & $25.5(17-33)$ & $32(20-50)$ & 0.70 \\
\hline AST, U/L & $30(11-47)$ & $31(13-45)$ & $33.2(12-49)$ & $37.1(17-51)$ & 0.57 \\
\hline Creatinine, mg/dl & $0.9(0.8-1.3)$ & $0.9(0.7-1.2)$ & $0.9(0.7-1.3)$ & $0.9(0.8-1.5)$ & 0.42 \\
\hline $\mathrm{LDH}, \mathrm{U} / \mathrm{L}$ & $446(325-571)$ & $380.3(325-574)$ & $465(333-574)$ & $326(286-491)$ & 0.14 \\
\hline $\mathrm{CK}, \mathrm{U} / \mathrm{L}$ & $113(71-278)$ & $113(81-345)$ & 113(71-345) & $85(64-158)$ & 0.57 \\
\hline Ferritin, ng/ml & 469(274-1141) & 462(274-1114) & 474(234-1141) & 407(337-1278) & 0.66 \\
\hline D-Dimer, ng/ml & $462(340-872)$ & 462(340-1076) & 437(340-804) & 413(340-799) & 0.96 \\
\hline $\mathrm{CRP}, \mathrm{mg} / \mathrm{dl}$ & $101(66-137)$ & $101(68-149)$ & $101(68-149)$ & $98(54-125)$ & 0.55 \\
\hline $\mathrm{PT}, \mathrm{s}$ & $14.7(13.5-16)$ & 14.6(13.5-16) & 14.65(13.4-15.9) & 14.9(14.1-16) & 0.94 \\
\hline Procalcitonin, ng/mL & $0.17(0.01-0.29)$ & $0.18(0.09-0.24)$ & $0.22(0.1-0.29)$ & $0.24(0.1-0.44)$ & 0.69 \\
\hline
\end{tabular}

Values were presented as median and interquartile range for non-normally distributed variables, and frequency and percentage for nominal variables

Abbreviations ALT: Alanine amino transferase, AST: Aspartate amino transferase, COPD: Chronic obstructive pulmonary disease, CK: Creatinine phosphokinase, CRP: protein, ICU: Intensive care unit, LDH: Lactate dehydrogenase, PT: Prothrombin time, WBC: White blood cell 
Table 2. Radiological findings of the patients

\begin{tabular}{|c|c|c|c|c|c|}
\hline & $\begin{array}{l}\text { All patients } \\
(n=130)\end{array}$ & $\begin{array}{l}\text { Patients who on } \\
\text { the ward until dis- } \\
\text { charge } \\
(n=75)\end{array}$ & $\begin{array}{l}\text { Patients who trans- } \\
\text { ferred to the ICU } \\
\qquad(n=38)\end{array}$ & $\begin{array}{l}\text { Patients who died } \\
\text { either on the ward } \\
\text { or in the ICU } \\
(n=17)\end{array}$ & $p$ \\
\hline $\begin{array}{l}\text { Type of Pulmoner } \\
\text { Involvement }\end{array}$ & & & & & 0.46 \\
\hline Consolidation & $36(27.7 \%)$ & $23(30.7 \%)$ & $11(28.9 \%)$ & $2(11.8 \%)$ & \\
\hline $\begin{array}{l}\text { Ground glass } \\
\text { appearance }\end{array}$ & $46(35.3 \%)$ & $25(33.3 \%)$ & $15(39.5 \%)$ & $6(35.3 \%)$ & \\
\hline $\begin{array}{l}\text { Bilateral } \\
\text { involvement }\end{array}$ & $48(36.9 \%)$ & $27(36 \%)$ & $12(31.6 \%)$ & $9(52.9 \%)$ & \\
\hline Psoas CSA, $\mathrm{cm}^{2}$ & 2.09 (1.55-3.06) & $2.55(1.74-3.44)$ & $1.96(1.67-2.62)$ & $1.35(1.17-1.6)$ & $<0.001$ \\
\hline $\mathrm{PMI}, \mathrm{cm}^{2} / \mathrm{kg} / \mathrm{m}^{2}$ & $0.09(0.07-0.12)$ & $0.11(0.08-0.14)$ & $0.09(0.08-0.11)$ & $0.06(0.05-0.07)$ & $<0.001$ \\
\hline Psoas density, HU & $49.9(44.6-53.7)$ & $50.20(43.84-54.52)$ & $50.27(44.74-53.23)$ & $48.9(46.94-50.49)$ & 0.587 \\
\hline
\end{tabular}

Values were presented as median and interquartile range for non-normally distributed variables, and frequency and percentage for nominal variables

Abbreviations CSA: Cross sectional area, ICU: Intensive care unit, PMI: Psoas muscle index

Table 3. Association between outcome measures, and clinical and radiological data

\begin{tabular}{|l|c|c|c|c|}
\hline & \multicolumn{2}{|c|}{ ICU admission } & \multicolumn{2}{c|}{ Mortality } \\
\hline & $p$ & Adj. OR (\%95 Cl) & Adj. OR (\%95 Cl) \\
\hline Age, years & 0.535 & $1.018(0.962-1.077)$ & 0.017 & 1.181 (1.030-1.355) \\
\hline Body temperature, ${ }^{\circ} \mathrm{C}$ & 0.514 & $0.956(0.836-1.094)$ & 0.746 & $0.961(0.757-1.221)$ \\
\hline Symptom Duration, days & 0.841 & $0.984(0.840-1.153)$ & 0.950 & $1.012(0.704-1.454)$ \\
\hline Comorbidity & 0.146 & $1.171(0.947-1.449)$ & 0.019 & $1.692(1.089-2.628)$ \\
\hline Psoas CSA, $\mathrm{cm}^{2}$ & 0.653 & $0.800(0.302-2.117)$ & 0.430 & $2.674(0.232-30.771)$ \\
\hline PMI, cm ${ }^{2} / \mathrm{kg} / \mathrm{m}^{2}$ & 0.845 & $0.860(0.195-3.796)$ & 0.001 & $\mathbf{5 . 2 0 4}(\mathbf{3 . 7 4 3 - 7 . 2 3 6 )}$ \\
\hline Psoas Density, HU & 0.916 & $1.003(0.943-1.068)$ & 0.294 & $0.934(0.821-1.061)$ \\
\hline
\end{tabular}

Abbreviations CSA: Cross sectional area, ICU: Intensive care unit, PMI: Psoas muscle index 
or level of diversity to accurately identify the relationship between muscle quality and prognosis.

While there are some other studies investigating the effect of CT-derived muscle properties on prognosis in COVID-19 patients, the present study is the first to examine the effect of psoas muscle measurements on prognosis. Previous studies have examined the effect of the CT-derived properties of the paravertebral and pectoral muscles on outcome, but they have reported conflicting results (22-24). Feng et al. found that the CSA and index of the erector spina and multifidus muscles at the level of the T12 vertebra were not associated with the composite outcome measure, which was determined as mortality and critical illness, among patients who underwent thoracic CT due to COVID-19; however, muscle density was associated with the composite outcome measure in women, but not in men, similar to our study (22). In a study by Poilina et al., no association could be identified between the paravertebral muscles index at the level of the T12 vertebra and mortality, ICU admission, or the need for invasive mechanical ventilation (23). Ufuk et al., in turn, reported that the pectoralis major and minor muscles index was associated with poor prognosis, as in our study (24). These conflicting results can be attributed to methodological factors, such as differences in muscle characteristics, the outcome measurements, and the age, gender, and comorbidities of the study patients. In addition, muscle CSA measurements at the L2 level are known to be better correlated with total body muscle mass than the muscle CSA measurement at the T12 level (25), which makes our results more reliable. Reliable results can only be achieved if the correct muscle is evaluated at the correct level.

As also shown in the present study, advanced age and comorbidity are well known to be associated with mortality in COVID-19 patients (3-4), and age and comorbidity remain important factors for elderly male patients. Another natural outcome of the present study's sample is the high mortality rate, which can be affected by patient population-relat- ed factors, such as age, gender, comorbidities, hospitalization, presence of lung involvement, and hospital facilities (24). It is thus not surprising that our mortality rate differs from that of other studies.

The present study has several limitations. First, and perhaps most importantly, was the inclusion of only older male patients, which affects the results' generalizability. With that said, obtaining results specific to the group most prone to frailty associated with sarcopenia can be considered valuable. Second, the study was conducted in a single center-a tertiary university hospital. A third limitation is that only the radiographic properties of the muscles were evaluated in the study, although muscle function is as important as muscle mass and included in the definition of sarcopenia. Finally, all thoracic CT scans do not extend to the L2 level, which makes the assessment of the psoas muscle difficult in daily practice, and it is not known to what extent the psoas muscle CSA of an upper level reflects the total muscle mass.

In conclusion, the PMI was found to be significantly associated with mortality in elderly male patients with COVID-19. In elderly male patients who are hospitalized due to COVID-19, an assessment of the psoas muscle may provide additional information on the prognosis of the disease. There is a need for further multi-center clinical studies that evaluate the PMI and muscle function together, involve young and female patients, and assess the effect on prognosis of the disease in COVID-19 patients.

\section{Acknowledgements}

The authors wish to thank the COVID-19 patients from the Nigde Omer Halisdemir University Training and Research Hospital who participated in this study. The authors have no conflict of interest, no disclosures, no funding or grant was provided for the project, no financial benefit will be or has been provided to the authors. 


\section{REFERENCES}

1. World Health Organization. WHO coronavirus (COVID-2019) dashboard; 2021. [Internet]. Available from: https://covid19.who.int/. Accessed: 30.03.2021.

2. Yang $Y$, Luo $K$, Jiang $Y$ et al. The impact of frailty on COVID-19 outcomes: A systematic review and meta-analysis of 16 cohort studies. J Nutr Health Aging 2021; 25(5): 702-709. (PMID: 33949641).

3. Zheng Z, Peng F, Xu B et al. Risk factors of critical \& mortal COVID-19 cases: A systematic literature review and meta-analysis. J Infect 2020; 81(2): 16-25. (PMID: 32335169).

4. Bello-Chavolla OY, Bahena-López JP, Antonio-Villa NE et al. Predicting mortality due to SARS-CoV-2: A mechanistic score relating obesity and diabetes to COVID-19 outcomes in Mexico. J Clin Endocrinol Metab 2020; 105(8): 2752-61. (PMID: 32474598).

5. National Institute for Health and Care Excellence. NICE updates rapid COVID-19 guideline on critical care; 2020. [Internet]. Available from: https://www. nice.org.uk/guidance/NG159. Accessed:01.06.2020.

6. Kara M, Kaymak B, Ata AM et al. STAR-sonographic thigh adjustment ratio: A golden formula for the diagnosis of sarcopenia. Am J Phys Med Rehabil 2020; 99(10): 902-908. (PMID: 32941253).

7. Cruz-Jentoft AJ, Bahat G, Bauer J et al. Sarcopenia: Revised European consensus on definition and diagnosis. Age Ageing 2019; 48(1): 16-31. (PMID: 30312372)

8. Wang PY, Li Y, Wang Q. Sarcopenia: An underlying treatment target during the COVID-19 pandemic. Nutrition 2021; 84: 104-111. (PMID: 33421827).

9. Teigen LM, Kuchnia AJ, Nagel E et al. Impact of software selection and ImageJ tutorial corrigendum on skeletal muscle measures at the third lumbar vertebra on computed tomography scans in clinical populations. JPEN J Parenter Enteral Nutr 2018; 42(5): 933-941. (PMID: 30001463).

10. Yoo T, Lo WD, Evans DC. Computed tomography measured psoas density predicts outcomes in trauma. Surgery 2017; 162(2): 377-384. (PMID: 28551380).

11. Akkoc I, Toptas M, Yalcin M et al. Psoas muscle area measured with computed tomography at admission to intensive care unit: Prediction of in-hospital mortality in patients with pulmonary embolism. Biomed Res Int 2020; 7(1): 158-164. (PMID: 32219127).
12. Moisey LL, Mourtzakis M, Cotton BA et al. Skeletal muscle predicts ventilator-free days, ICU-free days, and mortality in elderly ICU patients. Crit Care 2013; 17(5): 1-8. (PMID: 24050662).

13. The Republic of Turkey Ministry of Health. COVID-19 Patient Management Algorithm; 2020. [Internet]. Available from: https://covid19.saglik.gov. tr/TR-66926/eriskin-hasta-tedavisi.html. Accessed: 15.04.2020.

14. Zhou F, Yu T, Du R et al. Clinical course and risk factors for mortality of adult inpatients with COVID-19 in Wuhan, China: A retrospective cohort study. Lancet 2020; 395(10229): 1054-62. (PMID: 32171076)

15. Mourtzakis M, Prado CM, Lieffers JR, Reiman $T$, McCargar LJ, Baracos VE. A practical and precise approach to quantification of body composition in cancer patients using computed tomography images acquired during routine care. Appl Physiol Nutr Metab 2008; 33(5): 997-1006. (PMID: 18923576).

16. Izumi T, Watanabe J, Tohyama T, Takada Y. Impact of psoas muscle index on short-term outcome after living donor liver transplantation. Turk J Gastroenterol 2016; 27(4): 382-388. (PMID: 27458855).

17. Lo WD, Evans DC, Yoo T. Computed tomography-measured psoas density predicts outcomes after enterocutaneous fistula repair. JPEN J Parenter Enteral Nutr 2018; 42(1): 176-185. (PMID: 29505144).

18. Castaneda C, Charnley JM, Evans WJ, Crim MC. Elderly women accommodate to a low-protein diet with losses of body cell mass, muscle function, and immune response. Am J Clin Nutr 1995; 62(1): 30-39. (PMID: 7598064).

19. Ohara DG, Pegorari MS, Oliveira Dos Santos NL et al. Cross-sectional study on the association between pulmonary function and sarcopenia in Brazilian community-dwelling elderly from the Amazon region. J Nutr Health Aging 2020; 24(2): 181-187. (PMID: 32003408).

20. Wang L, Yin L, Zhao Y et al. Muscle density, but not size, correlates well with muscle strength and physical performance. J Am Med Dir Assoc 2021; 22(4): 751-759. (PMID: 32768372).

21. Lu J, Zheng ZF, Li P et al. A novel preoperative skeletal muscle measure as a predictor of postoperative complications, long-term survival and tumor recurrence for patients with gastric cancer after radical gastrectomy. Ann Surg Oncol 2018; 25(2): 439-448. (PMID: 29181681). 
22. Feng Z, Zhao H, Kang $W$ et al. Association of paraspinal muscle measurements on chest computed tomography with clinical outcomes in patients with severe coronavirus disease 2019. J Gerontol A Biol Sci Med Sci. 2021; 76(3): 78-84. (PMID: 33355656).

23. Moctezuma-Velázquez P, Miranda-Zazueta G, Ortiz-Brizuela E et al. Low thoracic skeletal muscle area is not associated with negative outcomes in patients with COVID-19. Am J Phys Med Rehabil 2021; 100(5): 413-418. (PMID: 33587451).
24. Ufuk F, Demirci M, Sagtas E, Akbudak IH, Ugurlu E, Sari T. The prognostic value of pneumonia severity score and pectoralis muscle Area on chest CT in adult COVID-19 patients. Eur J Radiol 2020; 131: 109-116. (PMID: 32942198).

25. Derstine BA, Holcombe SA, Ross BE, Wang NC, Su GL, Wang SC. Skeletal muscle cutoff values for sarcopenia diagnosis using T10 to L5 measurements in a healthy US population. Sci Rep 2018; 8(1): 113-121. (PMID: 30054580). 